\title{
Bread crust thickness measurement using digital imaging and $L$ a b colour system
}

\begin{abstract}
A simple and new method was developed for the evaluation of baking process on bread quality through the measurement of bread crust thickness. By distinguishing the crust and crumb regions of bread, the system which uses digital imaging and the $\mathrm{L}$ a b colour system can predict bread crust thickness from the colour measurements of bread surface browning. Standard baking tests were conducted at different levels of temperature and time combinations to produce open breads with different crust thickness. The results show that the crust thickness which ranged from 6.02 to $9.00 \mathrm{~mm}$ has a negative relationship with each of the $L, a$, and $b$ values and a positive correlation with the total colour difference ( $q$ E) of bread crust. The data also demonstrated that crust thickness increases with the investigated baking temperatures of 185,195 , and $205{ }^{\circ} \mathrm{C}$ more significantly $(\mathrm{p}<0.0001)$ than baking times of 25,30 and $35 \min (\mathrm{p}<0.001)$.
\end{abstract}

Keyword: Bread; Crust thickness; Digital imaging; L a b values 